\title{
The local well-posedness of solutions for a nonlinear pseudo-parabolic equation
}

\author{
Shaoyong Lai", Haibo Yan and Yang Wang
}

\section{"Correspondence:}

laishaoy@swufe.edu.cn

Department of Mathematics,

Southwestern University of Finance

and Economics, Wenjiang, Chengdu

611130, China

\section{Springer}

\begin{abstract}
The local existence and uniqueness of solutions for a nonlinear pseudo-parabolic equation are established in the Sobolev space $C\left([0, T) ; H^{s}\left(R^{n}\right)\right) \cap C^{1}\left([0, T) ; H^{s-1}\left(R^{n}\right)\right)$ with $s>\frac{n}{2}$. In addition, we prove the global existence of solutions for two special cases of the equation.
\end{abstract}

MSC: $35 \mathrm{Q} 35 ; 35 \mathrm{Q} 51$

Keywords: local strong solution; well-posedness; nonlinear pseudo-parabolic equation

\section{Introduction}

The pseudo-parabolic equation possesses the form

$$
\frac{\partial u}{\partial t}-k \frac{\partial \triangle u}{\partial t}=\Delta u+u^{p}, \quad x \in R^{n}, t>0
$$

where constant $k>0, p>0$, and $\Delta=\sum_{1}^{n} \frac{\partial^{2}}{\partial x_{i}^{2}}$. If $k=0$, Eq. (1) becomes the heat equation with sources. If $k>0$, we call Eq. (1) as the pseudo-parabolic model (see Ting [1], Showalter and Ting [2]). The pseudo-parabolic equation has many important physical backgrounds such as the seepage of homogeneous fluids through a fissured rock [3], the unidirectional propagation of nonlinear dispersive long waves $[4,5]$ and the aggregation of populations [6] (where $u$ is the population density). Equation (1) is employed in the analysis of nonstationary processes in the area of semiconductors $[7,8]$, where the term $k \frac{\partial \Delta u}{\partial t}-\frac{\partial u}{\partial t}$ is regarded as the free electron density rate, term $\Delta u$ is regarded as the linear dissipation of the free charge current and $u^{p}$ is a source of free electron current. Equation (1) is also named a Sobolev type model or a Sobolev-Galpern type model [9].

The initial-boundary value problem and the initial problem for the linear pseudoparabolic equation were investigated in $[1,2,10]$ where the existence and uniqueness of solutions for the equation were established. Various dynamic properties of solutions for nonlinear pseudo-parabolic equations, including singular pseudo-parabolic equations and degenerate pseudo-parabolic equations can be found in [11-18]. It is worth to mention that Kaikina et al. [19] considered the superlinear case of the Cauchy problem for Eq. (1) with $p>1$ and showed the existence and uniqueness of the solutions. Furthermore, it was shown that the Cauchy problem for Eq. (1) has a unique global solution under the assumptions $p>1+\frac{2}{n}$ and sufficiently small initial value $u_{0}$. The existence, uniqueness,

(c) 2014 Lai et al.; licensee Springer. This is an Open Access article distributed under the terms of the Creative Commons Attribution License (http://creativecommons.org/licenses/by/4.0), which permits unrestricted use, distribution, and reproduction in any medium, provided the original work is properly credited. 
and comparison principle for mild solutions of Eq. (1) were established in Cao et al. [20] by whom the large time behavior of the solutions and the critical global existence exponent and the critical Fujita exponent for Eq. (1) were obtained.

In this work, we study the following nonlinear pseudo-parabolic equation:

$$
\frac{\partial u}{\partial t}-k \frac{\partial \triangle u}{\partial t}=\Delta u+\alpha u^{q}+\beta D f(u), \quad x \in R^{n}, t>0,
$$

where $q \geq 1$ is an integer, $\alpha$ and $\beta$ are constants, $f(u)$ is a polynomial with order $m$, $f(0)=0$, and $D=\sum_{1}^{n} \frac{\partial}{\partial x_{i}}$. When $\beta=0$, Eq. (2) reduces to Eq. (1). The existence and uniqueness of local solutions for Eq. (2) are established in the Sobolev space $C\left([0, T) ; H^{s}\left(R^{n}\right)\right) \cap$ $C^{1}\left([0, T) ; H^{s-1}\left(R^{n}\right)\right)$ with $s>\frac{n}{2}$. We find that the local solution in the space $H^{s}\left(R^{n}\right)$ blows up if and only if $\lim _{t \rightarrow T}\|u(t, \cdot)\|_{L^{\infty}\left(R^{n}\right)}=\infty$. For the space dimension $n=1$, assuming that the initial value $u_{0} \in H^{1}\left(R^{1}\right), \alpha<0$, and $p$ is an odd number, we find the global existence of solutions for Eq. (2). For the other case $n=1, p=1$, and initial value $u_{0} \in H^{1}(R)$, we also acquire the global existence result of solutions for Eq. (2).

The rest of this paper is organized as follows. The main results are stated in Section 2. Several lemmas and the proofs of main results are given in Section 3.

\section{Main results}

Firstly, we state some notations.

Let $L^{p}=L^{p}\left(R^{n}\right)(1 \leq p<+\infty)$ be the space of all measurable functions $h$ such that $\|h\|_{L^{p}}^{p}=\int_{R^{n}}|h(t, x)|^{p} d x<\infty$. We define $L^{\infty}=L^{\infty}\left(R^{n}\right)$ with the standard norm $\|h\|_{L^{\infty}}=$ $\inf _{m(e)=0} \sup _{x \in R^{n} \backslash e}|h(t, x)|$. For any real number $s, H^{s}=H^{s}\left(R^{n}\right)$ denotes the Sobolev space with the norm defined by

$$
\|h\|_{H^{s}}=\left(\int_{R^{n}}\left(1+|\xi|^{2}\right)^{s}|\hat{h}(t, \xi)|^{2} d \xi\right)^{\frac{1}{2}}<\infty
$$

where $\hat{h}(t, \xi)=\int_{R^{n}} e^{-i x \xi} h(t, x) d x$.

For $T>0$ and nonnegative number $s, C\left([0, T) ; H^{s}\left(R^{n}\right)\right)$ denotes the Frechet space of all continuous $H^{s}$-valued functions on $[0, T)$. We set $\Lambda=\left(1-\sum_{1}^{n} \frac{\partial^{2}}{\partial x_{i}^{2}}\right)^{\frac{1}{2}}$ and $\Theta=(1-k \Delta)^{\frac{1}{2}}$. For simplicity, throughout this article, we let $c$ denote any positive constant.

We consider the Cauchy problem for Eq. (2)

$$
\left\{\begin{array}{l}
\frac{\partial u}{\partial t}-k \frac{\partial \Delta u}{\partial t}=\Delta u+\alpha u^{q}+\beta D f(u), \quad x \in R^{n}, t>0, \\
u(0, x)=u_{0}(x), \quad x \in R^{n}
\end{array}\right.
$$

which is equivalent to

$$
\left\{\begin{array}{l}
\frac{\partial u}{\partial t}=-\frac{1}{k} u+\Theta^{-2}\left[\frac{u}{k}+\alpha u^{q}+\beta D f(u)\right], \quad x \in R^{n}, t>0, \\
u(0, x)=u_{0}(x), \quad x \in R^{n},
\end{array}\right.
$$

where $\Theta^{-2}$ is the inverse operator of $\Theta^{2}=1-k \Delta$.

Now, we give our main results for problem (3).

Theorem 2.1 Let $u_{0}(x) \in H^{s}\left(R^{n}\right)$ with $s>\frac{n}{2}$. Then the Cauchy problem (3) has a unique solution $u(t, x) \in C\left([0, T) ; H^{s}\left(R^{n}\right)\right) \cap C^{1}\left([0, T) ; H^{s-1}\left(R^{n}\right)\right)$ where $T$ is the maximum existence 
time. Moreover,

$$
\lim _{t \rightarrow T}\|u(t, \cdot)\|_{H^{s}\left(R^{n}\right)}=\infty
$$

if and only if

$$
\lim _{t \rightarrow T}\|u(t, \cdot)\|_{L^{\infty}\left(R^{n}\right)}=\infty
$$

For the case of space dimension $n=1$, we have the result.

Theorem 2.2 Let $n=1, u_{0} \in H^{1}(R)$ in system (3), and assume that $q$ is an odd number and $\alpha \leq 0$. Then problem (3) has a unique global solution $u(t, x)$ satisfying

$$
u(t, x) \in C\left([0, \infty) ; H^{s}(R)\right) \cap C^{1}\left([0, \infty) ; H^{s-1}(R)\right), \quad s>\frac{1}{2}
$$

Theorem 2.3 Let $n=1, q=1$, and $u_{0} \in H^{1}(R)$ in system (3). For any constants $\alpha$ and $\beta$, then problem (3) has a unique global solution $u(t, x)$ satisfying

$$
u(t, x) \in C\left([0, \infty) ; H^{s}(R)\right) \cap C^{1}\left([0, \infty) ; H^{s-1}(R)\right), \quad s>\frac{1}{2}
$$

\section{Several lemmas}

Lemma 3.1 Let $r$ and $\rho$ be real numbers such that $-r<\rho \leq r$. Then

$$
\begin{aligned}
& \|u v\|_{H^{\rho}\left(R^{n}\right)} \leq c\|u\|_{H^{r}\left(R^{n}\right)}\|v\|_{H^{\rho}\left(R^{n}\right)}, \quad \text { if } r>\frac{n}{2}, \\
& \|u v\|_{H^{r+\rho-1 / 2\left(R^{n}\right)}} \leq c\|u\|_{H^{r}\left(R^{n}\right)}\|v\|_{H^{\rho}\left(R^{n}\right)}, \quad \text { if } r<\frac{n}{2} .
\end{aligned}
$$

This lemma can be found in [21] or [22].

Lemma 3.2 (Kato and Ponce [23]) If $r \geq 0$, then $H^{r} \cap L^{\infty}$ is an algebra. Moreover,

$$
\|u v\|_{H^{r}\left(R^{n}\right)} \leq c\left(\|u\|_{L^{\infty}\left(R^{n}\right)}\|v\|_{H^{r}\left(R^{n}\right)}+\|u\|_{H^{r}\left(R^{n}\right)}\|v\|_{L^{\infty}\left(R^{n}\right)}\right),
$$

where $c$ is a constant depending only on $r$.

Lemma 3.3 Assume $u_{0} \in H^{s}\left(R^{n}\right)$ with $s>\frac{n}{2}$. Then problem (3) admits a unique local solution

$$
u(t, x) \in C\left([0, T) ; H^{s}(R)\right) \cap C^{1}\left([0, T) ; H^{s-1}(R)\right) .
$$

Proof For the first equation of problem (4), we have

$$
u=u_{0}+\int_{0}^{t}\left(-\frac{u}{k}+\Theta^{-2}\left[\frac{u}{k}+\alpha u^{q}+\beta D f(u)\right]\right) d t
$$

Letting functions $u$ and $v$ be in the closed ball $B_{M_{0}}(0)$ of radius $M_{0}>1$ about the zero function in $C\left([0, T] ; H^{s}\left(R^{n}\right)\right)$ and letting $\Gamma$ be the operator on the right-hand side of (5), 
for fixed $t \in[0, T]$, we get

$$
\begin{aligned}
& \| \int_{0}^{t}\left(-\frac{u}{k}+\Theta^{-2}\left[\frac{u}{k}+\alpha u^{q}+\beta D f(u)\right]\right) d t \\
& \quad-\int_{0}^{t}\left(-\frac{v}{k}+\Theta^{-2}\left[\frac{v}{k}+\alpha v^{q}+\beta D f(v)\right]\right) d t \|_{H^{s}} \\
& \leq T\left(\sup _{0 \leq t \leq T}\|u-v\|_{H^{s}\left(R^{n}\right)}+\sup _{0 \leq t \leq T}\left\|u^{q}-v^{q}\right\|_{H^{s}\left(R^{n}\right)}\right. \\
&\left.\quad+\sup _{0 \leq t \leq T}\|f(u)-f(v)\|_{H^{s}\left(R^{n}\right)}\right) .
\end{aligned}
$$

Using Lemma 3.1 derives

$$
\begin{aligned}
\| u^{q}- & v^{q} \|_{H^{s}\left(R^{n}\right)} \\
& =\left\|(u-v)\left(u^{q-1}+u^{q-2} v+\cdots+u v^{q-2}+v^{q-1}\right)\right\|_{H^{s}\left(R^{n}\right)} \\
& \leq\|u-v\|_{H^{s}\left(R^{n}\right)}\left\|\left(u^{q-1}+u^{q-2} v+\cdots+u v^{q-2}+v^{q-1}\right)\right\|_{H^{s}\left(R^{n}\right)} \\
& \leq c M_{0}^{q-1}\|u-v\|_{H^{s}\left(R^{n}\right)}
\end{aligned}
$$

and

$$
\|f(u)-f(v)\|_{H^{s}\left(R^{n}\right)} \leq c M_{0}^{m-1}\|u-v\|_{H^{s}\left(R^{n}\right)}
$$

From (5)-(8), we obtain

$$
\|\Gamma u-\Gamma v\|_{H^{s}} \leq \theta\|u-v\|_{H^{s}\left(R^{n}\right)},
$$

where $\theta=\max \left(c T M_{0}, c T M_{0}^{q-1}, c T M_{0}^{m-1}\right)$ and $c$ is independent of $T$. Choosing $T$ sufficiently small such that $\theta<1$, we know that operator $\Gamma$ is a contractive mapping. Applying the above inequality and (5) yields

$$
\|\Gamma u\|_{H^{s}\left(R^{n}\right)} \leq\left\|u_{0}\right\|_{H^{s}\left(R^{n}\right)}+\theta\|u\|_{H^{s}\left(R^{n}\right)} .
$$

Choosing $T$ sufficiently small such that $\theta M_{0}+\left\|u_{0}\right\|_{H^{s}}<M_{0}$, we know that $\Gamma$ maps $B_{M_{0}}(0)$ to itself. It follows from the contractive mapping principle that the mapping $\Gamma$ has a unique fixed point $u$ in $B_{M_{0}}(0)$. This completes the proof.

Lemma 3.4 Let function $u(t, x)$ be a solution of problem (3), $s \geq \frac{n}{2}$ and the initial value $u_{0}(x) \in H^{s}\left(R^{n}\right)$. For $r \in(0, s-1]$, there is a constant $c$ depending only on the coefficients of the first equation of system (3) such that

$$
\begin{aligned}
\int_{R}\left(\Lambda^{r+1} u\right)^{2} d x \leq & \int_{R}\left(\Lambda^{r+1} u_{0}\right)^{2} d x \\
& +c \int_{0}^{t}\left(1+\|u\|_{L^{\infty}\left(R^{n}\right)}^{q-1}+\|u\|_{L^{\infty}\left(R^{n}\right)}^{m-1}\right)\|u\|_{H^{r+1}\left(R^{n}\right)}^{2} d \tau .
\end{aligned}
$$


Proof Using $\Delta=-\Lambda^{2}+1$ and the Parseval equality gives rise to

$$
\int_{R} \Lambda^{r} u \Lambda^{r} \Delta u d x=-\int_{R}\left(\Lambda^{r+1} u\right) \Lambda^{r+1} u d x+\int_{R}\left(\Lambda^{r} u\right)^{2} d x
$$

For $r \in(0, s-1]$, applying $\left(\Lambda^{r} u\right) \Lambda^{r}$ on both sides of the first equation of system (3), noting the above equality and integrating the resultant equation with respect to $x$ by parts, we obtain the equation

$$
\begin{aligned}
\frac{1}{2} \frac{d}{d t} & {\left[\int_{R}\left(\left(\Lambda^{r} u\right)^{2}+k\left(\Lambda^{r} u_{x}\right)^{2}\right) d x\right] } \\
= & -\int_{R^{n}}\left(\Lambda^{r+1} u\right) \Lambda^{r+1} u d x+\int_{R^{n}}\left(\Lambda^{r} u\right)^{2} d x \\
& +\alpha \int_{R^{n}}\left(\Lambda^{r} u\right) \Lambda^{r}\left(u^{q}\right) d x+\beta \int_{R^{n}}\left(\Lambda^{r} u\right) \Lambda^{r} f(u) d x \\
= & I_{1}+I_{2}+I_{3}+I_{4} .
\end{aligned}
$$

For the terms $I_{1}$ and $I_{2}$, we have

$$
\left|I_{1}\right| \leq\|u\|_{H^{r+1}\left(R^{n}\right)}^{2}
$$

and

$$
\left|I_{2}\right| \leq\|u\|_{H^{r+1}\left(R^{n}\right)}^{2}
$$

For the terms $I_{3}$ and $I_{4}$, using Lemma 3.2 gives rise to

$$
\begin{aligned}
\left|I_{3}\right| & \leq\left\|\Lambda^{r} u\right\|_{L^{2}\left(R^{n}\right)}\left\|\Lambda^{r}\left(u^{q}\right)\right\|_{L^{2}\left(R^{n}\right)} \\
& \leq c\|u\|_{H^{r}\left(R^{n}\right)}\|u\|_{L^{\infty}\left(R^{n}\right)}^{q-1}\|u\|_{H^{r}\left(R^{n}\right)} \\
& \leq c\|u\|_{L^{\infty}\left(R^{n}\right)}^{q-1}\|u\|_{H^{r+1}\left(R^{n}\right)}^{2}
\end{aligned}
$$

and

$$
\begin{aligned}
\left|I_{4}\right| & \leq c\left\|\Lambda^{r} u\right\|_{L^{2}\left(R^{n}\right)}\left\|\Lambda^{r}[D f(u)]\right\|_{L^{2}\left(R^{n}\right)} \\
& \leq c\left\|\Lambda^{r} u\right\|_{L^{2}\left(R^{n}\right)}\left\|\Lambda^{r+1} f(u)\right\|_{L^{2}\left(R^{n}\right)} \\
& \leq c\|u\|_{H^{r}\left(R^{n}\right)}\left(1+\|u\|_{L^{\infty}\left(R^{n}\right)}^{m-1}\right)\|u\|_{H^{r+1}\left(R^{n}\right)} \\
& \leq c\left(1+\|u\|_{L^{\infty}\left(R^{n}\right)}^{m-1}\right)\|u\|_{H^{r+1}\left(R^{n}\right)^{n}}^{2} .
\end{aligned}
$$

It follows from (12)-(16) that

$$
\begin{aligned}
& \frac{1}{2} \int_{R}\left[\left(\Lambda^{r} u\right)^{2}+k\left(\Lambda^{r} u_{x}\right)^{2}\right] d x-\frac{1}{2} \int_{R}\left[\left(\Lambda^{r} u_{0}\right)^{2}+k\left(\Lambda^{r} u_{0 x}\right)^{2}\right] d x \\
& \quad \leq c \int_{0}^{t}\left(1+\|u\|_{L^{\infty}\left(R^{n}\right)}^{q-1}+\|u\|_{L^{\infty}\left(R^{n}\right)}^{m-1}\right)\|u\|_{H^{r+1}}^{2} d \tau
\end{aligned}
$$

which results in (11). 
Proof of Theorem 2.1 Using Lemma 3.4, for any $s>\frac{n}{2}$, we have

$$
\|u\|_{H^{s}\left(R^{n}\right)} \leq c\left\|u_{0}\right\|_{H^{s}\left(R^{n}\right)} e^{\int_{0}^{t}\left[1+\|u\|_{L^{\infty}\left(R^{n}\right)}^{q-1}+\|u\|_{L^{\infty}\left(R^{n}\right)}^{m-1}\right.} d t
$$

For $s>\frac{n}{2}$, the Sobolev imbedding theorem yields

$$
\|u\|_{L^{\infty}\left(R^{n}\right)} \leq c\|u\|_{H^{s}\left(R^{n}\right)}
$$

Applying the inequalities (17), (18), and Lemma 3.3 completes the proof.

Proof of Theorem 2.2 For the space dimension $n=1$, we write problem (3) in the form

$$
\left\{\begin{array}{l}
u_{t}-k u_{t x x}=u_{x x}+\alpha u^{q}+\beta[f(u)]_{x}, \quad x \in R, t>0 \\
u(0, x)=u_{0}(x), \quad x \in R
\end{array}\right.
$$

Using $\int_{R} u^{j} u_{x} d x=0$ for any integer $j$ and integration by parts, we have

$$
\begin{aligned}
& \frac{1}{2} \int_{R} u^{2} d x \\
& \quad=\int_{R} u u_{t} d x \\
& \quad=\int_{R} u\left[k u_{t x x}+u_{x x}+\alpha u^{q}+\beta[f(u)]_{x}\right] d x \\
& \quad=\int_{R}\left[-k u_{x} u_{t x}-u_{x}^{2}+\alpha u^{q+1}\right] d x,
\end{aligned}
$$

which results in

$$
\frac{1}{2} \frac{d}{d t} \int_{R}\left(u^{2}+k u_{x}^{2}\right) d x+\int_{R} u_{x}^{2} d x-\alpha \int_{R} u^{q+1} d x=0,
$$

from which we obtain

$$
\frac{1}{2} \int_{R}\left(u^{2}+k u_{x}^{2}\right) d x+\int_{0}^{t} \int_{R}\left[u_{x}^{2}-\alpha u^{q+1}\right] d x d t=\frac{1}{2} \int_{R}\left(u_{0}^{2}+k u_{0 x}^{2}\right) d x
$$

If $q$ is an odd integer, $\alpha \leq 0$, and $u_{0} \in H^{1}(R)$, we get

$$
\|u(t, \cdot)\|_{L^{\infty}(R)} \leq c\left\|u_{0}\right\|_{H^{1}(R)} .
$$

Using the conclusion of Theorem 2.1, we finish the proof of Theorem 2.2.

Proof of Theorem 2.3 For $n=1$ and $q=1$, using (22) yields

$$
\frac{1}{2} \int_{R}\left(u^{2}+k u_{x}^{2}\right) d x+\int_{0}^{t} \int_{R}\left[u_{x}^{2}-\alpha u^{2}\right] d x d t=\frac{1}{2} \int_{R}\left(u_{0}^{2}+k u_{0 x}^{2}\right) d x
$$

Since

$$
\left|\int_{R}\left[u_{x}^{2}-\alpha u^{2}\right] d x\right| \leq(1+|\alpha|)\|u\|_{H^{1}(R)}^{2}
$$


it follows from (24) and (25) that

$$
\|u\|_{H^{1}(R)}^{2} \leq\left\|u_{0}\right\|_{H^{1}(R)}^{2} e^{(1+|\alpha|) t},
$$

\section{from which we obtain}

$$
\|u\|_{L^{\infty}(R)} \leq\left\|u_{0}\right\|_{H^{1}(R)} e^{(1+|\alpha|) t}
$$

which together with Theorem 2.1 completes the proof of Theorem 2.3.

\section{Competing interests}

The authors declare that they have no competing interests.

\section{Authors' contributions}

The article is a joint work of three authors who contributed equally to the final version of the paper. All authors read and approved the final manuscript.

\section{Acknowledgements}

This work is supported by both the Fundamental Research Funds for the Central Universities (JBK120504) and the Applied and Basic Project of Sichuan Province (2012JY0020).

\section{Received: 4 April 2014 Accepted: 1 July 2014 Published online: 24 September 2014}

\section{References}

1. Ting, TW: Parabolic and pseudo-parabolic partial differential equations. J. Math. Soc. Jpn. 21, 440-453 (1969)

2. Showalter, RE, Ting, TW: Pseudoparabolic partial differential equations. SIAM J. Math. Anal. 1, 1-26 (1970)

3. Barenblat, G, Zheltov, I, Kochiva, I: Basic concepts in the theory of seepage of homogeneous liquids in fissured rocks. J. Appl. Math. Mech. 24(5), 1286-1303 (1960)

4. Benjamin, TB, Bona, JL, Mahony, JJ: Model equations for long waves in nonlinear dispersive systems. Philos. Trans. R. Soc. Lond. Ser. A 272, 47-78 (1972)

5. Ting, TW: Certain non-steady flows of second-order fluids. Arch. Ration. Mech. Anal. 14, 1-26 (1963)

6. Padron, V: Effect of aggregation on population recovery modeled by a forward-backward pseudoparabolic equation. Trans. Am. Math. Soc. 356, 2739-2756 (2004)

7. Korpusov, MO, Sveshnikov, AG: Three-dimensional nonlinear evolution equations of pseudoparabolic type in problems of mathematical physics. Zh. Vychisl. Mat. Mat. Fiz. 43(12), 1835-1869 (2003)

8. Korpusov, MO, Sveshnikov, AG: Blow-up of solutions of Sobolev-type nonlinear equations with cubic sources. Differ Uravn. 42, 404-415 (2006)

9. Sobolev, SL: On a new problem of mathematical physics. Izv. Akad. Nauk SSSR, Ser. Mat. 18, 3-50 (1954)

10. Gopala, RVR, Ting, TW: Solutions of pseudo-heat equations in the whole space. Arch. Ration. Mech. Anal. 49, 57-78 (1972)

11. Brill, H: A semilinear Sobolev evolution equation in a Banach space. J. Differ. Equ. 24, 412-425 (1977)

12. David, C, Jet, W: Asymptotic behaviour of the fundamental solution to the equation of heat conduction in two temperatures. J. Math. Anal. Appl. 69, 411-418 (1979)

13. Karch, G: Asymptotic behaviour of solutions to some pseudoparabolic equations. Math. Methods Appl. Sci. 20, 271-289 (1977)

14. Karch, G: Large-time behaviour of solutions to nonlinear wave equations: higher-order asymptotics. Math. Methods Appl. Sci. 22, 1671-1697 (1999)

15. Ruggieri, M, Speciale, MP: Approximate analysis of a nonlinear dissipative model. Acta Appl. Math. (2014). doi:10.1007/s10440-014-9924-x

16. Kwek, KH, Qu, CC: Alternative principle for pseudo-parabolic equations. Dyn. Syst. Appl. 5, 211-217 (1996)

17. Levine, HA: Some nonexistence and instability theorems for solutions of formally parabolic equations of the form $P u_{t}=-A u+F(u)$. Arch. Ration. Mech. Anal. 51, 371-386 (1973)

18. Ptashnyk, M: Degenerate quasilinear pseudoparabolic equations with memory terms and variational inequalities. Nonlinear Anal. 66, 2653-2675 (2007)

19. Kaikina, El, Naumkin, PI, Shishmarev, IA: The Cauchy problem for a Sobolev type equation with power like nonlinearity. Izv. Math. 69, 59-111 (2005)

20. Cao, Y, Yin, JX, Wang, CP: Cauchy problems of semilinear pseudo-parabolic equations. J. Differ. Equ. 246, 4568-4590 (2009)

21. Kato, T: Quasi-linear equations of evolution with applications to partial differential equations. In: Spectral Theory and Differential Equations. Lecture Notes in Math., vol. 448, pp. 25-70. Springer, Berlin (1975)

22. Lai, SY, Wu, M: The local strong and weak solutions to a generalized Novikov equation. Bound. Value Probl. 2013 , Article ID 134 (2013)

23. Kato, T, Ponce, G: Commutator estimates and the Euler and Navier-Stokes equations. Commun. Pure Appl. Math. 41, 891-907 (1998) 
doi:10.1186/s13661-014-0177-4

Cite this article as: Lai et al.: The local well-posedness of solutions for a nonlinear pseudo-parabolic equation. Boundary Value Problems 2014 2014:177.

Submit your manuscript to a SpringerOpen ${ }^{\circ}$ journal and benefit from:

- Convenient online submission

- Rigorous peer review

- Immediate publication on acceptance

- Open access: articles freely available online

- High visibility within the field

- Retaining the copyright to your article

Submit your next manuscript at $\gg$ springeropen.com 